\title{
Advancing molecular imaging: a chairman's perspective on how radiology can meet the challenge
}

\author{
Hedvig Hricak
}

Received: 26 April 2010 / Accepted: 13 June 2010 / Published online: 31 August 2010

(C) Springer-Verlag 2010

\begin{abstract}
To date, most molecular imaging techniques applied clinically have offered relatively general information about the metabolism and physiology of diseased cells and tissues. However, due to recent scientific and technological advances, much more specifically targeted molecular imaging probes (e.g., reporter gene probes, whole cell-tracking probes, and probes for localizing specific biomolecules) are now being used in preclinical research and, in some cases, translated to the clinical setting. As a result, the imaging community is poised to help lead a revolution in personalized, molecularly targeted medicine. This article considers the importance of molecular imaging for advancing research and clinical care both within individual institutions and across the medical field. It outlines specific steps that leaders in academic radiology can take to hasten progress in molecular imaging and explains why they must have the courage to reach across traditional interdisciplinary boundaries and advocate for major investments in equipment, education, and personnel.
\end{abstract}

Keywords Molecular imaging · Personalized medicine . Small-animal imaging $\cdot$ Biomarkers $\cdot$ Leadership

Cross-sectional imaging transformed radiology into an essential guiding hand of medical practice. In parallel with this, rapid biological advances, such as the sequencing of the human genome and the explosion of innovations in

H. Hricak $(\bowtie)$

Department of Radiology,

Memorial Sloan-Kettering Cancer Center,

1275 York Avenue,

New York, NY 10065, USA

e-mail: muellnea@mskcc.org proteinomics, have made this the age of molecular medicine. Now, thanks to advances in molecular imaging, the radiology community has a chance to help lead a revolution in medicine toward optimal, personalized diagnosis and therapy.

Molecular imaging allows in-vivo detection, characterization and quantitative analysis of key molecules, molecular events and cellular components that are fundamental to the pathophysiology of human disease [1-6]. Imaging techniques that fit this description have been in use for a number of years and include contrast-enhanced MRI, MR spectroscopic imaging, single photon emission CT (SPECT), positron emission tomography (PET) and optical imaging, among others [7-12]. To date, most molecular imaging techniques applied clinically have provided information about general aspects of the metabolism (in large part glucose metabolism) and physiology of diseased cells and tissues, such as neovascularization (altered blood volume, permeability, perfusion and vascularity). However, thanks to scientific and technological advances, probes for more specifically targeted molecular imaging are now being tested in animals and, in some cases, translated to the clinical setting. These include targeted probes for localizing specific biomolecules, such as signal transduction proteins or tumor-associated antigens; reporter gene probes, which allow monitoring of the actions of genes in living biological systems; and whole-cell tracking probes, which are used to localize and follow the movement of cells, such as cancer cells, inflammatory cells or stromal cells [13, 14].

The clinical use of more specific molecular imaging probes, along with high-throughput serum screening and biologically targeted biopsies, will make it possible to apply a growing array of imaging, serum and tissue biomarkers to detect and characterize disease in humans. These will include predictive biomarkers for identifying the relative sensitivity or resistance of a disease to a specific therapy, or 
for identifying disease not in need of treatment; biomarkers for assessing treatment response earlier than is possible with conventional means; and prognostic biomarkers that provide information about the likely patient outcome regardless of the specific treatment applied [15]. In addition, molecular imaging will make possible the increasing use of theranostics - i.e. combinations of targeted diagnostic and therapeutic agents that work sequentially or in tandem. As discussed elsewhere in this special issue of Pediatric Radiology, the result is expected to be a paradigm shift from one-size-fits-all to personalized, molecular medicine. However, many obstacles stand in the way of this transition; overcoming them will require unprecedented levels of interdisciplinary collaboration as well as bold leaders willing to make substantial investments in equipment, education and personnel.

The infrastructure to support molecular imaging should now be considered essential for any modern medical research facility. This infrastructure must include resources for small-animal imaging. Animal models of disease make it possible to perform certain kinds of studies that are difficult, if not impossible, to perform in humans [16]. The greatest advantage of noninvasive animal imaging is the ability to repetitively observe the biological processes underlying disease. Not only does animal imaging expand our scientific knowledge and facilitate improvements in imaging technology, it is absolutely critical for developing new molecular imaging agents that can eventually be applied in humans. Industry has responded to the growing demand for small-animal imaging equipment and the devices now available include micro-MR, micro-CT, micro-SPECT, micro-PET, micro-US and various devices for optical imaging.

The National Institutes of Health (NIH) roadmap includes initiatives to facilitate molecular imaging research, including the creation of an Imaging Probe Development Center. Furthermore, the National Cancer Institute has provided funding for Small Animal Imaging Resource Programs (SAIRPs) and In Vivo Cellular and Molecular Imaging Centers (ICMICs) at selected academic institutions [17-20]. The latter facilitate molecular imaging research specifically for cancer and bring together scientists and physicians from numerous fields, including molecular and cellular biology, radiochemistry, medical physics, nuclear medicine, diagnostic radiology, pharmacology, biomathematics and bioinformatics, among others. ICMICs have already shown that molecular imaging is a powerful engine of discovery: in 2009 , nine of the ten radiology departments receiving the most funding from the NIH had ICMICs or similar dedicated molecular imaging programs [21].

It is also essential to develop strategies for accelerating the clinical translation of new molecular imaging probes. In the next 10 years, a number of molecular imaging probes are expected to be approved including probes used for the imaging of metastatic lymph nodes, vulnerable atherosclerotic plaque, macrophages in inflammatory and degenerative diseases (e.g., multiple sclerosis, polyarthritis and osteomyelitis) and amyloid plaques in Alzheimer's disease. However, many more probes are on the horizon. At present, the expense of clinical development and the regulatory process are the major rate-limiting factors in probe development.

The design of molecular imaging probes must take into account pharmacokinetics, biocompatibility and toxicity on the one hand, and imaging modality sensitivity, speed and resolution on the other. The development process usually produces numerous candidates, of which only a few pass preclinical evaluation with the promise of clinical utility. The most suitable substances have to undergo in-depth toxicological evaluation before clinical trials can even begin. Furthermore, the use of experimental molecular imaging techniques in hospital settings is often difficult due to the expense of the multimodality imaging equipment, the lack of reimbursement and the lack of personnel with the expertise needed to develop, apply and interpret the new techniques. Collaboration between academia, industry, medical societies and government regulators can help to address these problems and streamline probe development.

Even the most sophisticated infrastructure for research and clinical practice in molecular imaging will be of little value without personnel who are properly trained to use it. I am convinced that, as radiologists, the single greatest challenge we will face in meeting the demands of molecular medicine will be to recruit and train a cadre of young physicians with the drive and knowledge to become innovators and leaders in the field of molecular imaging. To capture the interest of medical students, we must work to create rotations that provide early exposure to the combination of biology and technology which is at the heart of this discipline. Radiology training programs should encourage participation in a fellowship component to deepen scientific competence, and, in particular, more molecular imaging fellowships should be offered. We must begin to create a new breed of radiologists - specialists in molecular imaging - who will be trained in both diagnostic imaging and nuclear medicine and possess a solid understanding of physics, radiochemistry and biology. Such specialists will also need to be prepared to carry out the molecular imaging components of theranostic procedures, particularly for treating cancer.

In oncology today, one of the most critical challenges is the detection, characterization and treatment of metastatic disease. Molecular imaging is the only diagnostic tool that can be used to detect and assess the heterogeneous biology of all metastatic sites simultaneously, and for this reason it is expected to play an especially important role in the 
clinical care of cancer patients. At present, however, few radiology residencies include training in oncology and very few oncological imaging fellowships or continuing medical education programs in oncological imaging are available worldwide. If molecular imaging and theranostics are to reach their full potential, these educational gaps must be addressed.

The key tasks for leaders in academic radiology will include the following:

- Identify leaders and mentors who are interested in advancing modern, molecular medicine and are willing to learn and grow with the field. The mentors are needed to educate young physician scientists who will carry radiology forward and ensure the continued relevance of our profession.

- Identify, train, encourage and support the young faculty of the future who will advance molecular imaging and continue to change the way radiology is practiced.

- Work on impacting the national educational boards of radiology to realize where the future lies and revamp training curricula to make them more timely and relevant.

- Supply modern infrastructure with equipment and wet laboratory space and ensure funding for continuous operational expenses, training and faculty support.

- Deepen our partnerships with the critical allied scientists who are coworkers in the development of radiology and particularly molecular imaging. These include imaging physicists, radiochemists, nanotechnology engineers, etc.

As the chair of an academic radiology department within a large dynamic cancer centre, I have been impressed with how rapidly the landscape of medicine is changing. As physicians of the 21st Century, we live in revolutionary times and like it or not, we have become participants in the era of molecular medicine. The opportunities for radiology as a specialty have never been greater, and I have no doubt that great rewards as well as great risks lie ahead. From the standpoint of our field, I feel certain that we can meet the challenges of this exciting but uncertain future. Molecular imaging will clearly be a driving engine for academic growth and the development of revolutionary improvements in patient care. Our task as leaders is to build and maintain a community of trainees, clinician scientists imaging scientists and chemists who can lead this revolution.
Acknowledgements The author thanks Ada Muellner, MS for editing this manuscript.

\section{References}

1. Hillman BJ, Neiman HL (2002) Translating molecular imaging research into radiologic practice: summary of the proceedings of the American College of Radiology Colloquium, April 22-24, 2001. Radiology 222:19-24

2. Jaffer FA, Weissleder R (2005) Molecular imaging in the clinical arena. JAMA 293:855-862

3. Jager PL, de Korte MA, Lub-de Hooge MN et al (2005) Molecular imaging: what can be used today. Cancer Imaging 5: S27-32

4. Pomper MG (2001) Molecular imaging: an overview. Acad Radiol 8:1141-1153

5. Pomper MG (2005) Translational molecular imaging for cancer. Cancer Imaging 5:S16-26

6. Weissleder R, Mahmood U (2001) Molecular imaging. Radiology 219:316-333

7. Choy G, Choyke P, Libutti SK (2003) Current advances in molecular imaging: noninvasive in vivo bioluminescent and fluorescent optical imaging in cancer research. Mol Imaging $2: 303-312$

8. Gambhir SS (2002) Molecular imaging of cancer with positron emission tomography. Nat Rev Cancer 2:683-693

9. Hicks RJ (2003) Beyond FDG: novel PET tracers for cancer imaging. Cancer Imaging 4:22-24

10. Hicks RJ (2005) The role of PET in monitoring therapy. Cancer Imaging 5:51-57

11. Hicks RJ, Ware RE, Lau EW (2006) PET/CT: will it change the way that we use CT in cancer imaging? Cancer Imaging 6:S52-62

12. Luker GD, Piwnica-Worms D (2001) Molecular imaging in vivo with PET and SPECT. Acad Radiol 8:4-14

13. Kiessling F (2008) Noninvasive cell tracking. Handb Exp Pharmacol 185:305-321

14. Serganova I, Mayer-Kukuck P, Huang R et al (2008) Molecular imaging: reporter gene imaging. Handb Exp Pharmacol 185:167223

15. (2010) The promise of imaging biomarkers. Drug News Perspect 23:119-123

16. Hoffman JM, Croft BY (2001) Future directions in small animal imaging. Lab Anim (NY) 30:32-35

17. Clarke LP, Croft BY, Menkens A et al (2000) National Cancer Institute initiative for development of novel imaging technologies. Acad Radiol 7:481-483

18. Hoffman JM (2000) Imaging in cancer: a National Cancer Institute "extraordinary opportunity." Neoplasia 2:5-8

19. Hoffman JM, Gambhir SS (2007) Molecular imaging: the vision and opportunity for radiology in the future. Radiology 244:39-47

20. Hoffman JM, Menkens AE (2000) Molecular imaging in cancer: future directions and goals of the National Cancer Institute. Acad Radiol 7:905-907

21. Academy of Radiology Research. NIH rankings of grants to radiology departments. Available at: http://www.acadrad.org/. Accessed April 26, 2010 